\title{
A FRAMEWORK FOR MALICIOUS AGENT DETECTION IN CLOUD COMPUTING ENVIRONMENT
}

\author{
Ishu Gupta ${ }^{1 *}$ and Ashutosh Kumar Singh ${ }^{2}$ \\ Department of Computer Applications, National Institute of Technology, \\ Kurukshetra, Haryana, India \\ ${ }^{1}$ ishugupta23@gmail.com, ${ }^{2}$ ashutosh@nitkkr.ac.in
}

\begin{abstract}
Data security is one of the major challenges encountered by cloud computing. The cloud data is shared among multiple entities which can be intentionally or unintentionally revealed out by any agent to the unauthorized recipient. Therefore, it has become a necessity to detect the malicious agent for protecting shared information. In this regard, we present a framework based on the probabilistic estimation that identifies the malicious agent for minimizing the likelihood of further leakage. In the proposed model, the data is distributed among multiple agents and the allocation is performed using 2-level trees. The parameters based on probability theory are computed for malicious agent identification when the data is leaked by any agent. The experimental results achieved average probability, average success rate, and detection rate up to $1,0.98,0.76$ respectively for the various number of agents.
\end{abstract}

Keywords - Data Leakage, Information Security, Leaker Detection, Threat Model, Probabilistic Approach

\section{INTRODUCTION}

Cloud computing is gaining popularity among industries, academia, government and business communities due to its on demand facilities. It provides hardware infrastructure and application oriented software on pay per use basis which significantly minimizes the capital investment. The key features of cloud computing are flexibility, disaster recovery, automatic software and hardware updates, work from anywhere, security and competitiveness etc. [1-3]. Because of these features, around $77 \%$ of the enterprises are using the cloud services all over the world for various applications [4]. Data sharing in the cloud environment is an essential step among various stakeholders to elevate their business performances. However, the users or agents who receive the data for different purposes may misuse or leak this data that can cause a heavy loss to the various enterprises in terms of finance and reputation [5]. As a consequence, data leakage has become a major challenge in securing the cloud data [6-8]. According to a survey conducted by Gemalto breach level index, 4.5 billion records have been exposed worldwide due to 945 data breaches in the first half of 2018 which shows a rise of $133 \%$ compared to the same period in previous year [9].

The methods that deal with the malicious agent detection can be classified into two broad categories (i) watermarking (ii) probability based approaches. In watermarking [10-15] based methods, before handing over the data (text, image, audio, video, relational etc.) to

Received: August 21, 2019

Reviewed: January 12, 2020

Accepted: February 12, 2020

* Corresponding Author 
the agent, a secret code is embedded in the transferable document using watermark embedding process. If the critical data leaked by the agent is discovered from unauthorized place; malicious agent is detected by extracting the embedded watermark from the leaked document using watermark extraction process. But, if the watermarked is destroyed or tempered in the embedded data by malicious agent, then the leaker can't be identified [16]. However, the probability method is not affected by the aforementioned problem raised in watermarking based approaches and identify the leaker on the basis of allocated data. Different methods [16-20] has been proposed to distribute the data among agents during allocation process and several parameters are evaluated for the guilty agent identification. The proposed model allocates the shared data using the 2-level trees and detects the malicious agent responsible for leakage.

Rest of the paper is organized as follows: Section 2 provides the brief view of the proposed model along with basic definitions, symbols and threat model. Section 3 and 4 discuss about the data allocation and malicious agent detection in detail. The performance is supported by the numerous experimental analysis in Section 5 followed by conclusion in Section 6.

\section{PROPOSED MODEL}

The threat model used in the proposed framework consists three different entities data owner $\mathrm{O}^{\mathrm{D}}$, cloud server $\mathrm{C}^{\mathrm{S}}$, and agent $\mathrm{A}_{\mathrm{j}}$ where data confidentiality is considered as the most serious threat. The entity agent is considered as an attacker and the objective of the model is to identify the malicious agent that leaks the data to the unauthorized third party. In addition to this, the action can be taken against the guilty party. Following are the basic definitions used for the proposed model:

\section{Data Owner}

A data owner $\mathrm{O}^{\mathrm{D}}$ possesses the data $\mathrm{D}=\left\{\mathrm{D}_{1}, \mathrm{D}_{2}, \ldots \mathrm{Dn}\right\}$ to be stored in the cloud server $\mathrm{C}^{\mathrm{S}}$ and is accountable for the distribution of $\mathrm{D}$. $\mathrm{O}^{\mathrm{D}}$ has to allocate $\mathrm{D}$ among various agents $\left.\mathrm{A}=\mathrm{A}_{1}, \mathrm{~A}_{2}, \ldots, \mathrm{A}_{\mathrm{m}}\right\}$ with the intention that data must not be leaked by the agent. It is considered as a trusted party in the model.

Agents

An agent $\mathrm{A}_{\mathrm{j}}$ acquires the data $Y_{j} \subseteq \mathcal{D}$ by requesting it from $\mathrm{O}^{\mathrm{D}}$ and carry out required tasks via utilizing $\mathrm{Y}_{\mathrm{j}}$. This entity is intended as an untrusted party in the model.

\section{Data Leakage}

Accidentally or advertently disclosure of private or sensitive information to the unauthorized party is termed as data leakage. The sensitive or private data in the enterprises include financial, personal, medical information etc.

\section{Malicious Agent}

The entity is named as malicious agent $\mathrm{M}_{\mathrm{A}}$ that leaked the data $D^{L} \subseteq D$ from its allocated dataset $\mathrm{Y}_{\mathrm{j}}$ to the unauthorized third party which can misuse $\mathrm{D}^{\mathrm{L}}$.

\section{Third Party}

An entity that maliciously obtains the data and may maltreat is named as a third party. It belongs to the model in an indirect manner.

\section{Cloud Server}

The entity having considerable $\mathrm{D}$ to be stored that is provisioned by $\mathrm{O}^{\mathrm{D}}$ named as cloud server $\mathrm{C}^{\mathrm{S}}$. The stored data is shared among $A_{j} \forall j=1,2, \ldots, m$; on their demands.

The complete process for malicious agent $\mathrm{M}_{\mathrm{A}}$ detection is shown in Fig. 1. The requests are obtained from multiple agents $A_{j} ; j=\{1,2, \ldots, m\}$ and the data owner $\mathrm{O}^{\mathrm{D}}$ allocates the data to the agent $\mathrm{A}_{\mathrm{j}}$ The data allocation is performed using the 2-level trees 
$T_{1}(A, D, E)$ and $T_{2}(D, A, E)$ in the model. If intended $\mathrm{A}_{\mathrm{j}}$ leaks the allocated data $\mathrm{Y}_{\mathrm{j}}$ either intentionally or unintentionally, then responsible $\mathrm{M}_{\mathrm{A}}$ is to be recognized by applying the detection mechanism. The proposed model has two major steps: (i) data allocation and (ii) probability evaluation which are discussed in subsequent sections in detail. All the used notations and their corresponding descriptions are tabulated in Table I.

Table I.

\begin{tabular}{|c|l|}
\hline Notation & \\
\hline$O^{D}$ & Data owner \\
\hline$D_{i}$ & Data object \\
\hline$A_{j}$ & An agent \\
\hline$Y_{j}$ & Allocated dataset to agent $A_{j}$ \\
\hline$M_{A}$ & Malicious agent \\
\hline$D^{L}$ & Leaked dataset \\
\hline$C^{5}$ & Cloud server \\
\hline$T(U, V, E)$ & 2-level tree \\
\hline$D_{i}^{\prime}$ & Encrypted document \\
\hline$\Delta_{\mathrm{A}_{j}}$ & Total number of request fulfilled of agent $A_{j}$ \\
\hline$X_{i}$ & Agent set among which $D_{i}$ is distributed \\
\hline$\Omega_{\mathrm{D}_{1}}$ & Total number of agents having $D_{i}$ in $Y_{j}$ \\
\hline$K$ & Key used for encryption \\
\hline$M_{A_{j}}$ & the event that agent $A_{j}$ is malicious \\
\hline$\Theta$ & Probability of guessing \\
\hline$\Psi^{*}$ & Average success rate \\
\hline$m i n \Psi^{*}$ & Detection rate \\
\hline
\end{tabular}

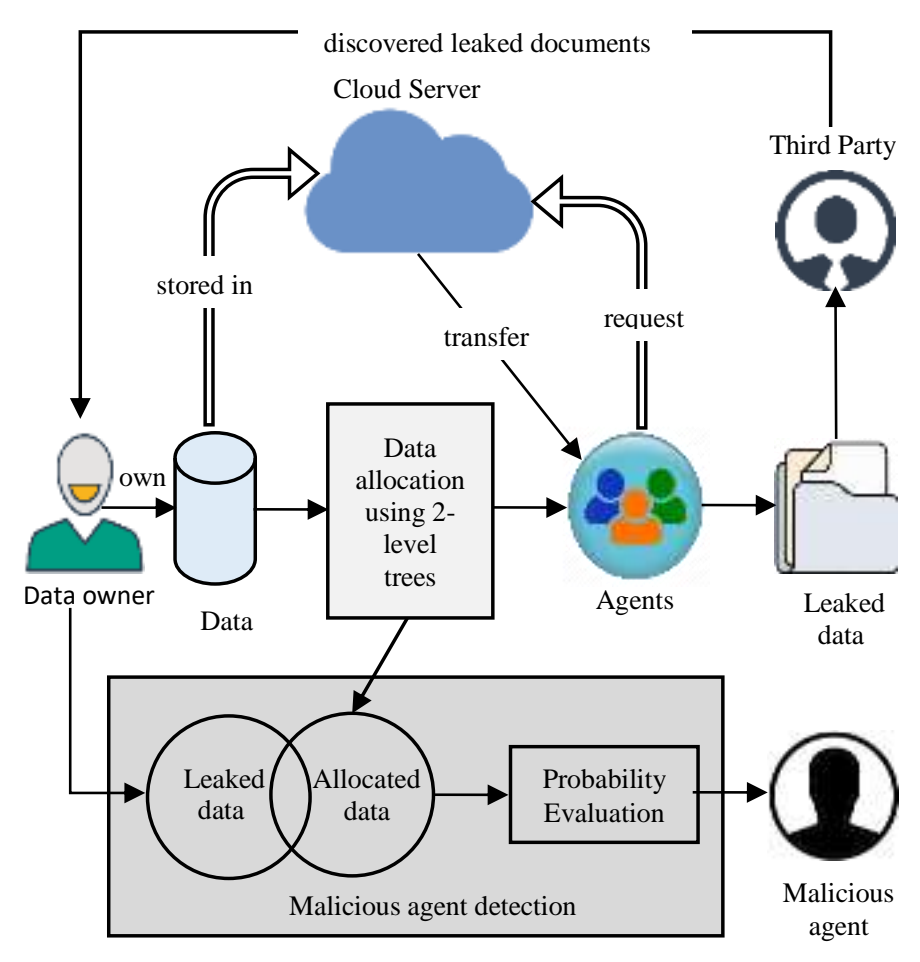

Fig. 1. Proposed Framework 


\section{DATA ALLOCATION}

Agent $A_{j}$ makes the request to $C^{S}$ for required document $D_{i} ; i \in\{1,2, \ldots, n\} . O^{D}$ verifies the previous record of $A_{j}$ as well as availability of $D_{i}$ in $C^{S}$. Once, $A_{j}$ is found trustworthy and $D_{i}$ is available, $O^{D}$ encrypts $D_{i}$ to ensure the more security to it and delivers the generated encrypted document $D_{i}^{\prime}$ to $A_{j}$. Similarly, the request of all $A_{j} \in A$ is fulfilled by $O^{D}$ after examining their malicious record and availability of required $D_{i} \in D$ retained in the database.

Data allocation among various agents $A_{j} \in A$ is implemented using 2-level Trees $T_{1}$ and $T_{2} . T(U, V, E)$ is a 2-level tree where $U$ is the root node, $V$ represents the leaf nodes and $E$ is the set of edges exist from $U$ to $V$. An edge $e \in E$ exists between the two nodes iff $T$ remains circuit free and connected. $T_{1}\left(A_{j}, D^{\prime}, E_{1}\right)$ provides the dataset $Y_{j}=D^{\prime} \subseteq D$ received by $A_{j} \in A$ as shown in Fig. 2(a). This figure shows that an edge exists between $A_{j}$ and $D_{i}$ i.e. $e_{j, i} \in E_{1}$ iff $A_{j}$ obtains the required $D_{i}$. The total number of requests accomplished of an agent $A_{j} \in A$ is given by $\Delta_{A_{j}}=\operatorname{deg}\left(A_{j}\right) . T_{2}\left(D_{i}, A^{\prime}, E_{2}\right)$ in Fig. 2(b) provides the allocation of $D_{i} \in D$ among the agents $A^{\prime} \subseteq A$. There exists an edge between data $D_{i}$ and an agent $A_{j}$ i.e. $e_{i, j} \in E_{2}$ iff data object $D_{i}$ is allocated to the agent $A_{j} . \mathrm{X}_{i}=$ $A^{\prime} \subseteq A$ is obtained from $T_{1}$ which is the set of agents having $D_{i}$ in their allocated dataset $Y_{j} . \Omega_{D_{i}}=\operatorname{deg}\left(D_{i}\right)$ gives the total number of agents to whom data object $D_{i}$ is allocated.

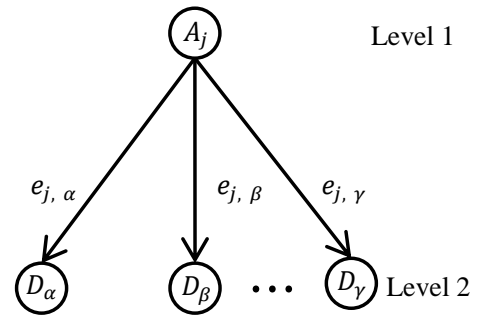

(a)

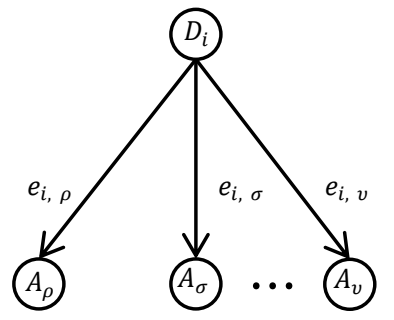

(b)

Fig. 2. 2-Level tree (a) $T_{1}\left(A_{j}, D^{\prime}, E_{1}\right)$ (b) $T_{2}\left(D_{i}, A^{\prime}, E_{2}\right)$

\section{Example 1}

Let the model consists of seven data objects $D=\left\{D_{1}, D_{2}, D_{3}, D_{4}, D_{5}, D_{6}, D_{7}\right\}$ and four agents $A=\left\{A_{1}, A_{2}, A_{3}, A_{4}\right\}$. The agents $A_{j} \forall j=1,2,3,4$; submit their requests for the required data objects to $C^{S}$. The data is distributed among agents as per their requests and the allocation is accomplished via $T_{1}$ and $T_{2}$. The allocated dataset to the agents $A_{1}, A_{2}, A_{3}, A_{4}$ are:

$\Upsilon_{1}=\left\{D_{2}, D_{4}, D_{5}, D_{6}, D_{7}\right\}$

$\Upsilon_{2}=\left\{D_{2}, D_{3}, D_{5}, D_{6}\right\}$

$\Upsilon_{3}=\left\{D_{1}, D_{3}, D_{5}\right\}$

$\Upsilon_{4}=\left\{D_{1}, D_{2}, D_{3}, D_{4}, D_{6}, D_{7}\right\}$

The allocation of $D_{1}, D_{2}, D_{3}, D_{4}, D_{5}, D_{6}, D_{7}$ among the agents are:

$X_{1}=\left\{A_{3}, A_{4}\right\}$,

$X_{2}=\left\{A_{1}, A_{2}, A_{4}\right\}$,

$X_{3}=\left\{A_{2}, A_{3}, A_{4}\right\}$,

$X_{4}=\left\{A_{1}, A_{4}\right\}$,

$X_{5}=\left\{A_{1}, A_{2}, A_{3}\right\}$,

$X_{6}=\left\{A_{1}, A_{2}, A_{4}\right\}$,

$X_{7}=\left\{A_{1}, A_{4}\right\}$ 
The request fulfilled of the agents $A_{1}, A_{2}, A_{3}, A_{4}$ are $\Delta_{A_{1}}=5, \Delta_{A_{2}}=4, \Delta_{A_{3}}=3$ and $\Delta_{A_{4}}=6$ respectively. The total number of agents to whom $D_{1}, D_{2}, D_{3}, D_{4}, D_{5}, D_{6}, D_{7}$ has given are $\Omega_{D_{1}}=2, \Omega_{D_{2}}=3, \Omega_{D_{3}}=3, \Omega_{D_{4}}=2, \Omega_{D_{5}}=3, \Omega_{D_{6}}=3$ and $\Omega_{D_{7}}=2$ respectively. The pseudo code for data distribution is shown in Algorithm 1.

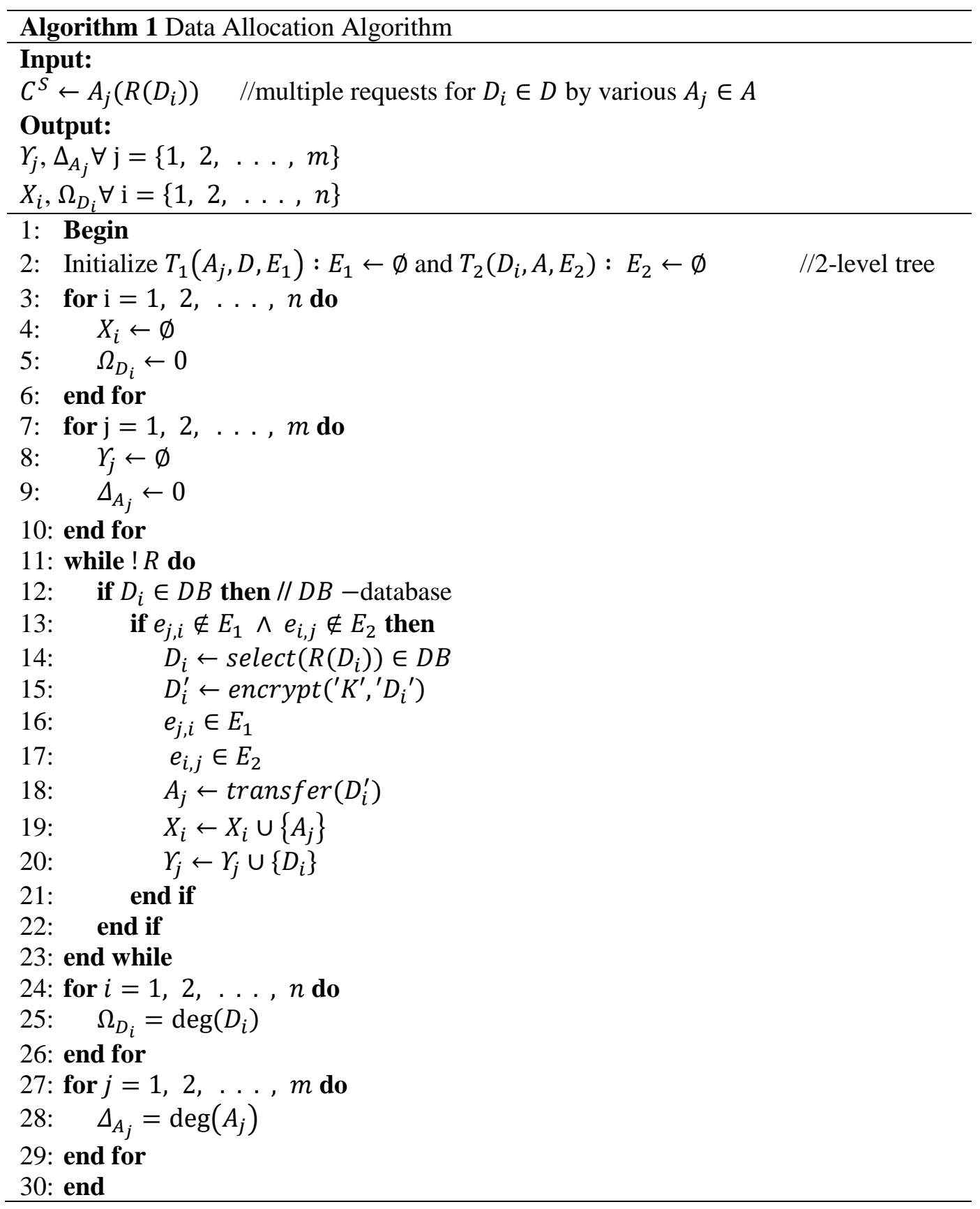

\section{MALICIOUS AGENT DETECTION}

Let $M_{A_{j}}$ is the event that agent $A_{j}$ is the malicious agent $M_{A}$. In our model, there are two possibilities for obtaining the data by target $t$ : (i) any single agent from the set $\mathrm{X}_{i}$ has leaked object $D_{i}$ to $t$ (ii) $t$ retrieved the data object $D_{i}$ by guess or through any other mean without intervention of any agent $A_{j}$. The probability to leak any data object $D_{i}$ is equal $\forall A_{j} \in \mathrm{X}_{i}$ if it is leaked by any agent $A_{j} \in X_{i}$, otherwise probability is $\Theta$ if it is obtained by $t$. It is 
considered that decision of $A_{j}$ to leak any data object $D_{i}$ is autonomous to the leaking of other data object $D_{i^{\prime}} \forall D_{i}, D_{i^{\prime}} \in D^{L}$ where $D_{i} \neq D_{i^{\prime}}$. For the given leaked dataset $D^{L}$, the conditional probability $P\left\{M_{A_{j}} \mid D^{L}\right\}$ of the agent $A_{j}$ to be malicious is computed by Eq. (1) where $\Theta$ is the probability of guessing the data object $D_{i}$ and $\Omega_{D_{i}}$ signifies the number of $A_{j}$ to whom the object $D_{i}$ has been alloacted.

$P\left\{M_{A_{j}} \mid D^{L}\right\}=1-\prod_{\forall D_{i} \in\left(D^{L} \cap r_{j}\right)}\left(1-\frac{(1-\Theta)}{\Omega_{D_{i}}}\right)$

If $A_{j}$ leaks all the data objects from its allocated set $Y_{j}$ such that $D^{L}=\Upsilon_{j}$, the probability $P\left\{M_{A_{j}} \mid Y_{j}\right\}$ of $A_{j}$ for being $M_{A}$ is computed. Difference function $\Psi_{(j, k)}^{*}\left(M_{A}\right)$ given in Eq. (2) maximizes the possibility of identifying $M_{A}$ which is obtained in the form of a $m \times m$ matrix.

$\Psi_{(j, k)}^{*}\left(M_{A}\right)=P\left\{M_{A_{j}} \mid \Upsilon_{j}\right\}-P\left\{M_{A_{k}} \mid \Upsilon_{j}\right\} \quad \forall j, k=\{1,2, \ldots, m\}$

To evaluate and analyze the performance of the proposed approach, two parameters $\bar{\Psi}^{*}$ and $\min \Psi^{*}$ are calculated with the help of the matrix $\Psi_{(j, k)}^{*}\left(M_{A}\right)$ in Eq. (3) and Eq. (4) respectively. $\bar{\Psi}^{*}$ is the average success rate which is computed by taking the average of all the entries of the matrix $\Psi_{(j, k)}^{*}\left(M_{A}\right) \cdot \min \Psi^{*}$ renders the detection rate which represents the minimum entry of the matrix $\Psi_{(j, k)}^{*}\left(M_{A}\right)$. Algorithm 2 depicts the pseudo code for probability computation.

$\bar{\Psi}^{*}=\frac{\sum_{\substack{j, k=\{1,2, \ldots, m\} \\ j \neq k}} \Psi_{(j, k)}^{*}\left(M_{A}\right)}{m(m-1)}$

$\min \Psi^{*}=\min _{\substack{j, k=\{1,2, \ldots, m\} \\ j \neq k}} \Psi_{(j, k)}^{*}\left(M_{A}\right)$

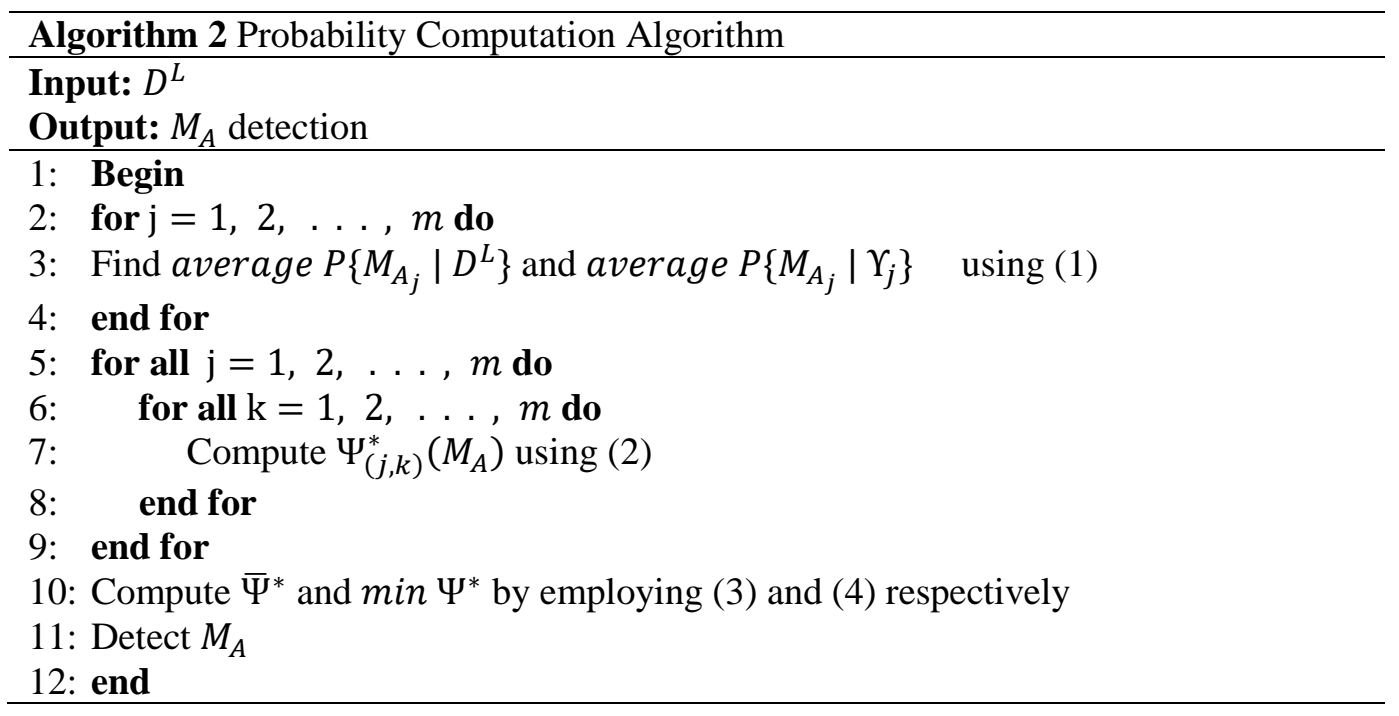

Let the leaked dataset $D^{L}=\left\{D_{2}, D_{4}, D_{5}, D_{6}, D_{7}\right\}$ is found at the unauthorized place in example 1, the probability of the agents $A_{1}, A_{2}, A_{3}, A_{4}$ are computed using Eq. (1), to estimate $M_{A}$ for $\Theta=0.1$. The values of computed probabilities are:

$P\left\{M_{A_{1}} \mid D^{L}\right\}=0.8962$,

$P\left\{M_{A_{2}} \mid D^{L}\right\}=0.657$

$P\left\{M_{A_{3}} \mid D^{L}\right\}=0.3$,

$P\left\{M_{A_{4}} \mid D^{L}\right\}=0.8518$ 
The probability of the agents $A_{1}, A_{2}, A_{3}, A_{4}$ to leak their dataset $\Upsilon_{1}, \Upsilon_{2}, \Upsilon_{3}, \Upsilon_{4}$ respectively are computed and given as:

$P\left\{M_{A_{1}} \mid Y_{1}\right\}=0.8962$,

$P\left\{M_{A_{1}} \mid Y_{1}\right\}=0.7599$,

$P\left\{M_{A_{3}} \mid Y_{3}\right\}=0.7305$,

$P\left\{M_{A_{4}} \mid \Upsilon_{4}\right\}=0.9429$

$\Psi_{(j, k)}^{*}\left(M_{A}\right)$ is evaluated for all $j, k=\{1,2,3,4\}$ using Eq. (2) as depicted in Matrix

छ. Furthermore $\bar{\Psi}^{*}=0.2344$ and $\min \Psi^{*}=0.0444$ are calculated using Eq. (3) and Eq. (4) respectively.

$$
\Xi=\left(\begin{array}{cccc}
0 & 0.2392 & 0.5962 & 0.0444 \\
0.1029 & 0 & 0.2499 & 0.1029 \\
0.4305 & 0.2205 & 0 & 0.1155 \\
0.0915 & 0.28593 & 0.32793 & 0
\end{array}\right)
$$

\section{PERFORMANCE EVALUATION}

The experiments are conducted using $\mathrm{C} / \mathrm{C}++$ on a machine equipped with Intel ${ }^{\circledR}$ coreTM I5 processor with $2.60 \mathrm{GHz}$ clock speed and $8 \mathrm{~GB}$ RAM. To analyze the performance, $|D|=500$ is considered while the numbers of agents vary for different circumstances. The performance of three parameters: (i) probabilities for $M_{A}$ detection (ii) average success rate (iii) detection rate have been evaluated against the weight factor $W_{F}$. Weight factor can be defined as the ratio of requests fulfilled of all the agents to the total number of data objects as given in Eq. (5).

$W_{F}=\frac{\sum_{j=1}^{m} \Delta_{A_{j}}}{|D|}$

The value of $W_{F}$ varies between 1 to 6 throughout the experiments. There are two possible ways to alter $W_{F}$ : (i) number of agents are unchanged but their request size change (ii) number of agents change with their fixed request size. Furthermore, the number of requests can be same or differ for all the agents. Therefore, three circumstances are arisen for the experimental analysis: (a) number of agents are fixed i.e. $|A|=40$ and request size changes in the range $(1-75)$ for different scenario while considering the same number of requests of all the agents (b) fixed number of agents i.e. $|A|=100$ with same request size in every scenario in the range $(1-30)$ (c) number of agents vary from 2 to 80 in all scenarios with its different request from the range $(30-50)$.

\subsection{AVERAGE PROBABILITY}

Fig. 3 shows the probability $P\left\{M_{A_{j}} \mid D^{L}\right\}$ for all three aforementioned circumstances when the leaked data set is given, where 250 data objects have been taken in $D^{L}$ for $\Theta=$ $0,0.25,0.5,0.75,0.9$. The distinct values of $\Theta$ and $W_{F}$ provide the different probabilities to identify $M_{A}$. It can be observed from Fig. 3(a, b) that probability increases with increment in $W_{F}$, but decreases with increment in $\Theta$. Furthermore, we notice that the probability to detect $M_{A}$ decays when the number of agents raise. However, in Fig 3(c), the probability reduces with augmentation in both $W_{F}$ and $\Theta$. 


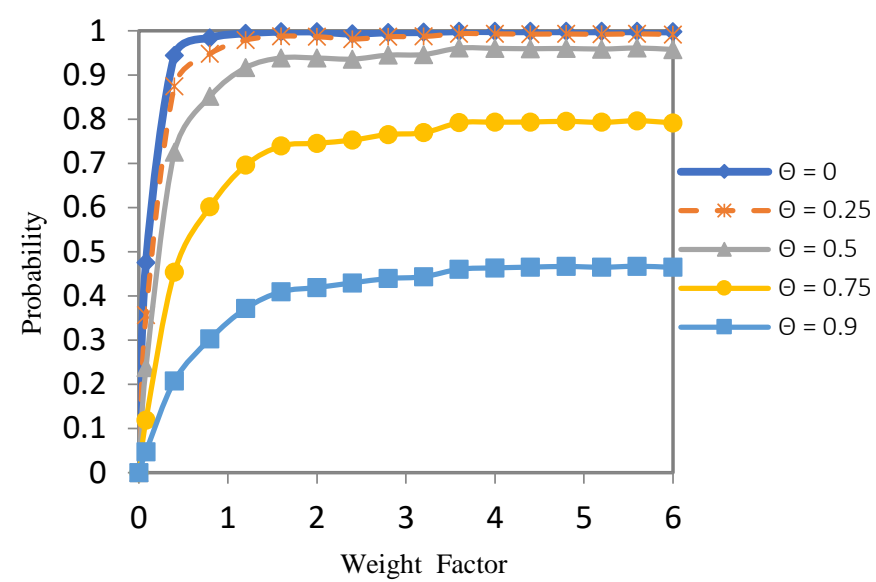

(a)

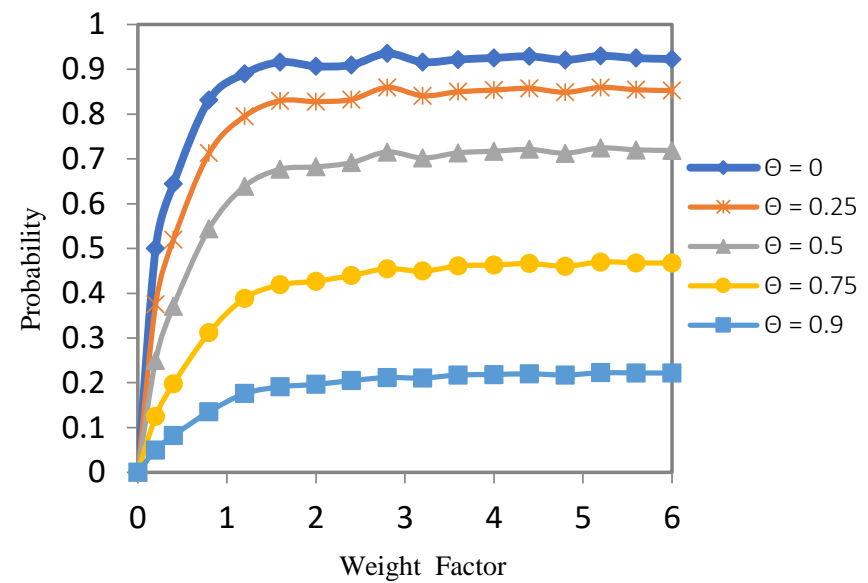

(b)

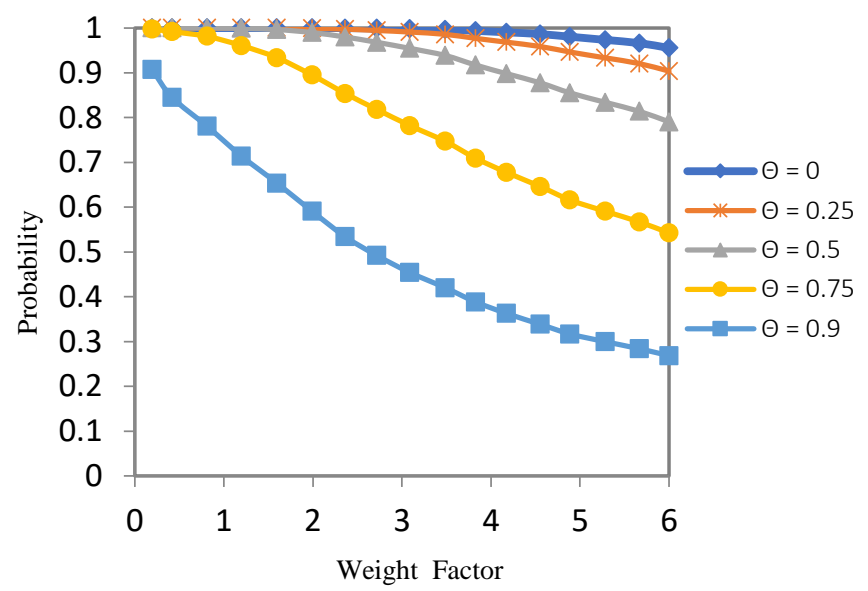

(c)

Fig. 3. Evaluation of $P\left\{M_{A_{j}} \mid D^{L}\right\}$ at $|L|=250$ (a) $|A|=40$ (b) $|A|=100$ (c) $|A|=$ $(2-80)$

Fig. 4 shows the probability $P\left\{M_{A_{j}} \mid D^{L}\right\}$ when the data in $D^{L}$ changes for the fixed value of $\Theta$. For all three aforementioned circumstances, $|L|=\{100,200,300,400\}$ and $\Theta=0.4$ has been considered. In this figure, the probability enhances with increment of data in $D^{L}$. 


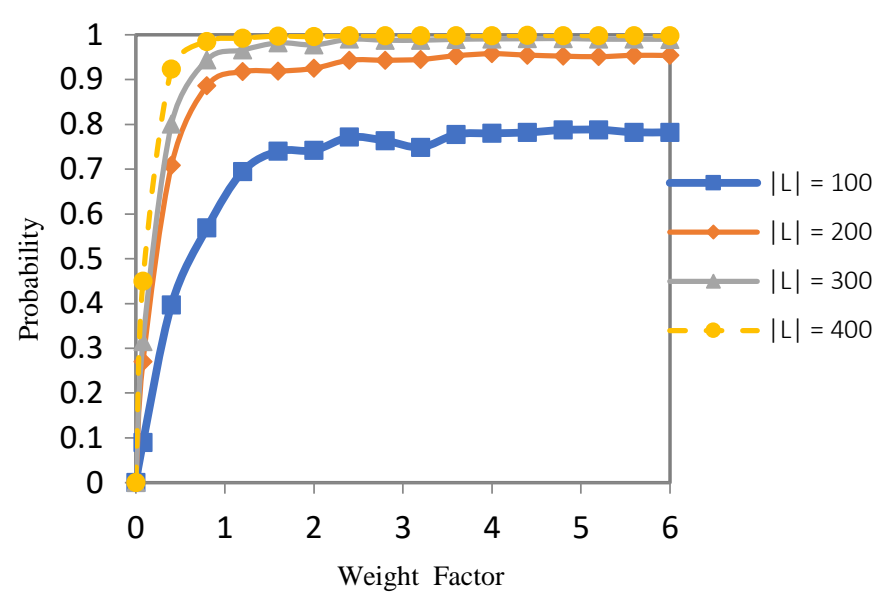

(a)

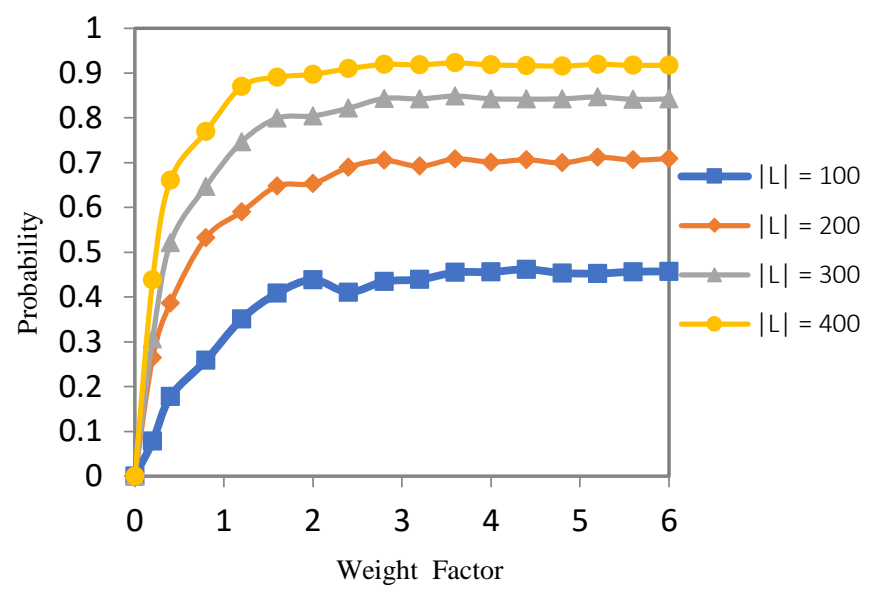

(b)

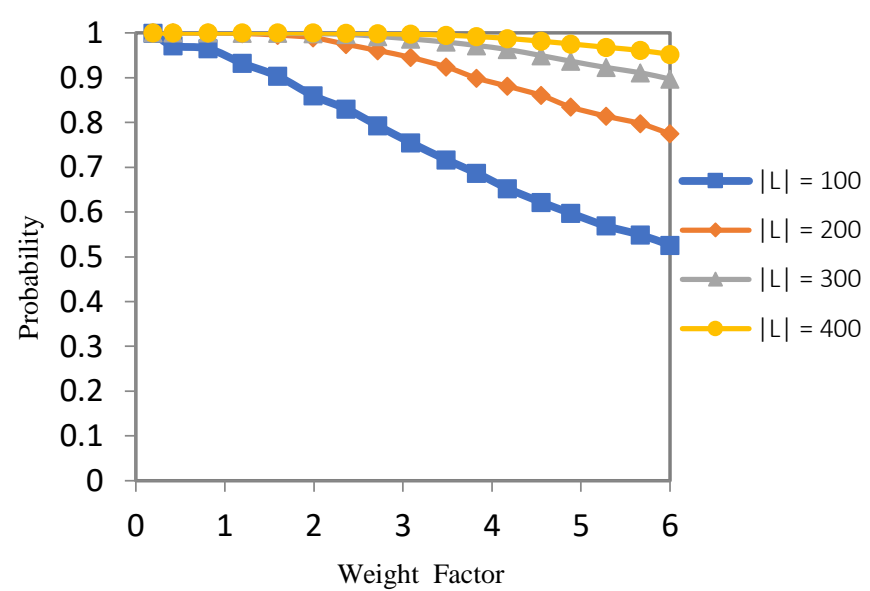

(c)

Fig. 4. Evaluation of $P\left\{M_{A_{j}} \mid D^{L}\right\}$ at $\Theta=0.4$ (a) $|A|=40$ (b) $|A|=100$ (c) $|A|=$ $(2-80)$

Fig. 5 represents the average probability $P\left\{\mathrm{M}_{\mathrm{A}_{\mathrm{j}}} \mid Y_{\mathrm{j}}\right\}$ when all the allocated dataset $Y_{\mathrm{j}}$ is leaked by all the agents. The probability to identify the malicious agent is high even for large value of $W_{F}$ and $\Theta$. 


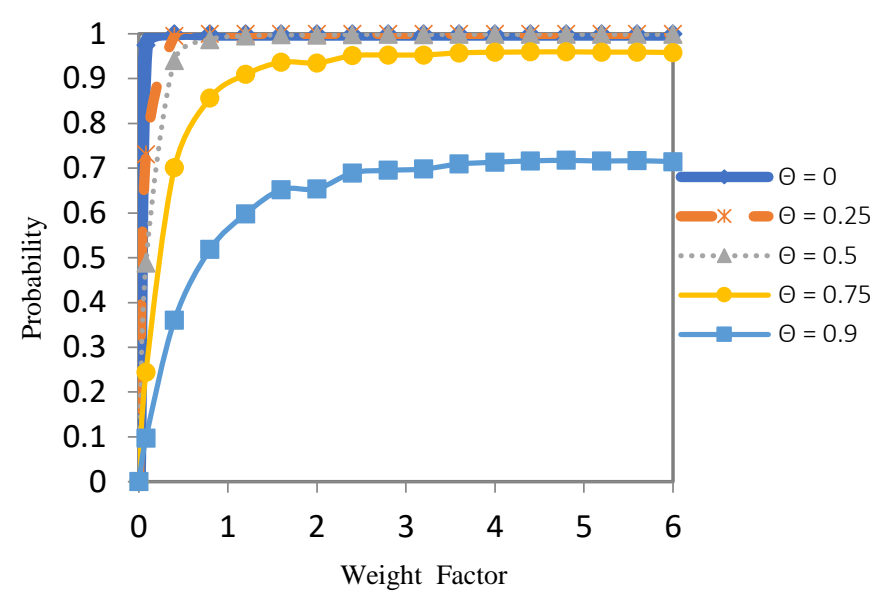

(a)

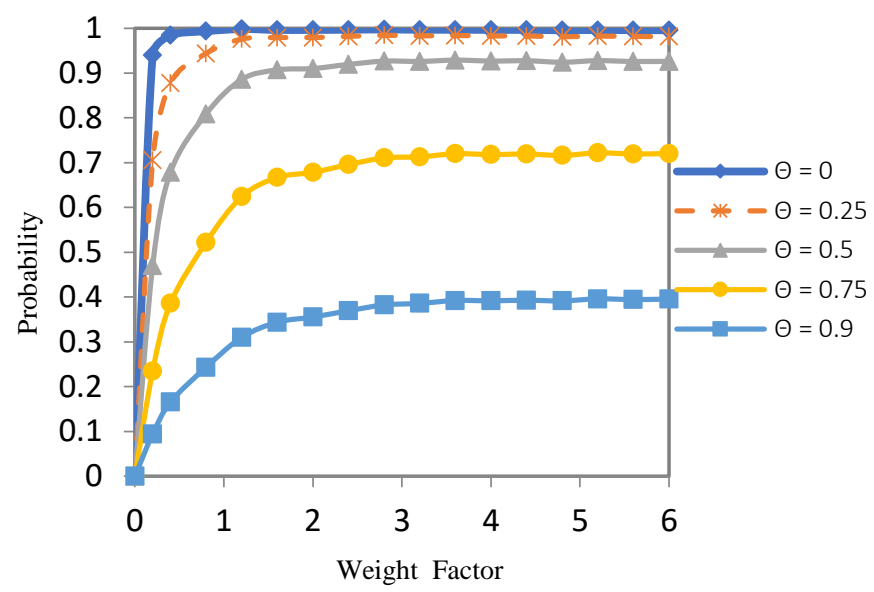

(b)

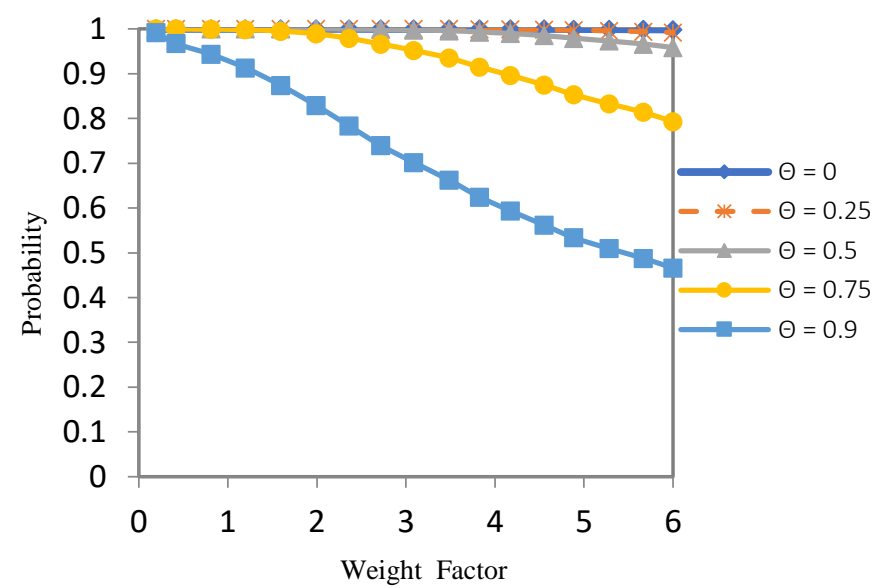

(c)

Fig. 5. Evaluation of $P\left\{M_{A_{j}} \mid Y_{j}\right\}$ (a) $|A|=40$ (b) $|A|=100$ (c) $|A|=(2-80)$

From Fig. (3-5), probability decays with increment of $\Theta$ in all the circumstances as chances of guessing the data increases. For the fixed number of agents in the model, probability rises with increment in $W_{F}$ as overlapping of allocated dataset minimizes. When the data in leaked dataset increases (Fig. $(4,5)$ ), the probability of detecting $M_{A}$ also escalates as we obtain more evidences against $M_{A}$. 


\subsection{AVERAGE SUCCESS RATE}

Fig. 6 portrays the parameter $\bar{\Psi}^{*}$ with respect to $\Theta$ and $W_{F}$. In Fig. 6 (a, b), it is noticed that $\bar{\Psi}^{*}$ initially increases and then decreases or remains constant with respect to $W_{F}$. Moreover, for larger number of agents, $\bar{\Psi}^{*}$ is lesser as depicted in Fig. 6 (b). In Fig. 6 (c), $\bar{\Psi}^{*}$ increases initially and then it starts decreasing with enhancement in $W_{F}$, but it increases for the lower value of $\Theta$.

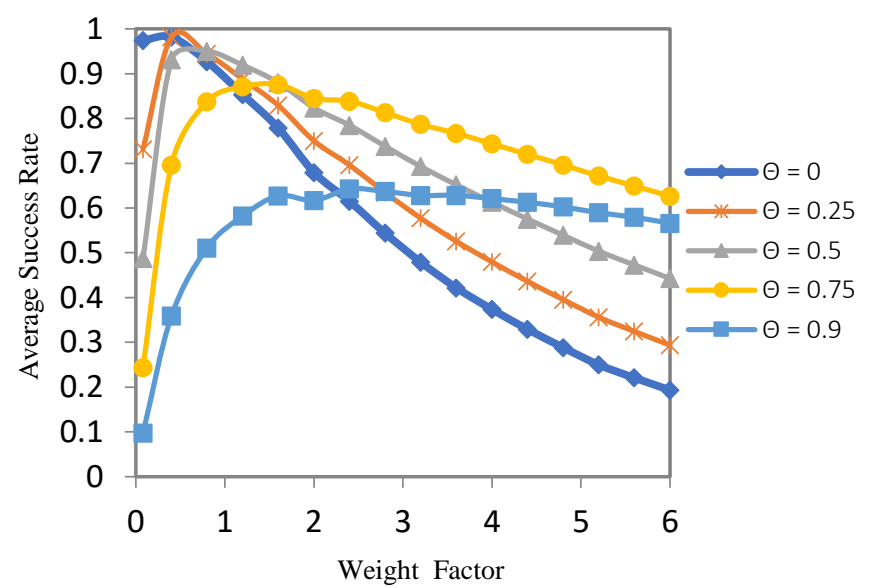

(a)

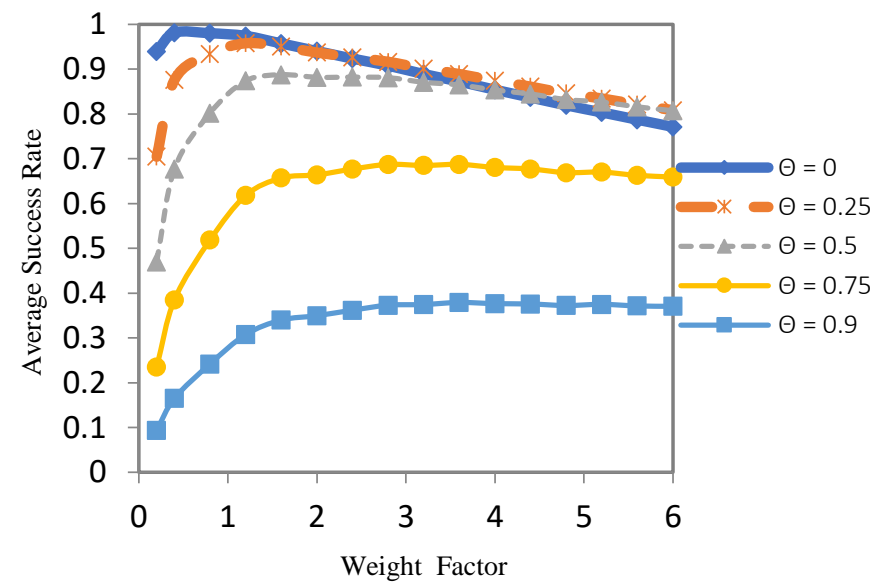

(b)

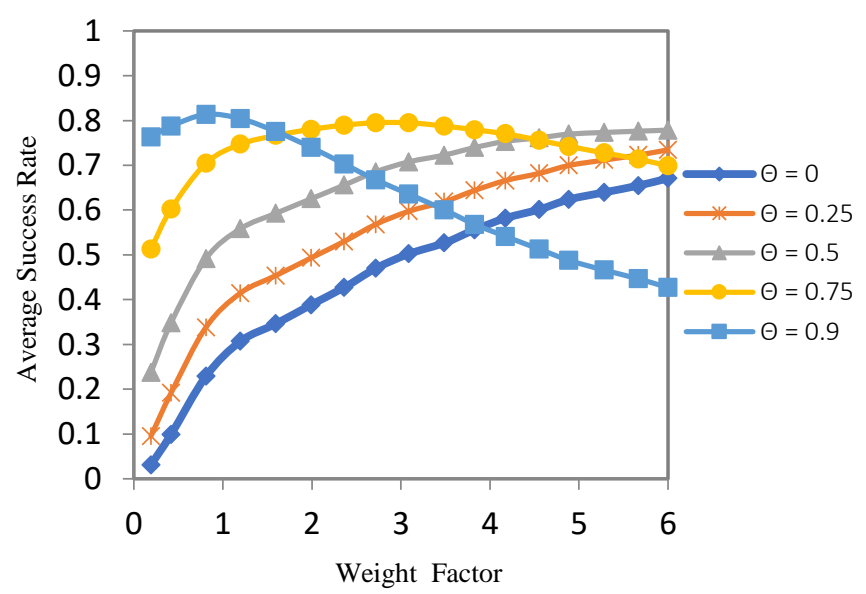

(c)

Fig. 6. Evaluation of $\bar{\Psi}^{*}$ (a) $|A|=40$ (b) $|A|=100$ (c) $|A|=(2-80)$ 


\subsection{DETECTION RATE}

Fig. 7 depicts the parameter $\min \Psi^{*}$ with respect to $W_{F}$ for different values of $\Theta$. In Fig. 7 (a), the detection rate decreases for $\Theta=0,0.25,0.5$ and increases or almost constant for $\Theta=0.75,0.9$ with increment in $W_{F}$. However, $\min \Psi^{*}$ keeps increasing for all $\Theta$ in Fig. 7 (b) because of large number of agents. In the last case (Fig. 7 (c)), $\min \Psi^{*}$ escalates with increment in $\Theta$ except $\Theta=0.9$.

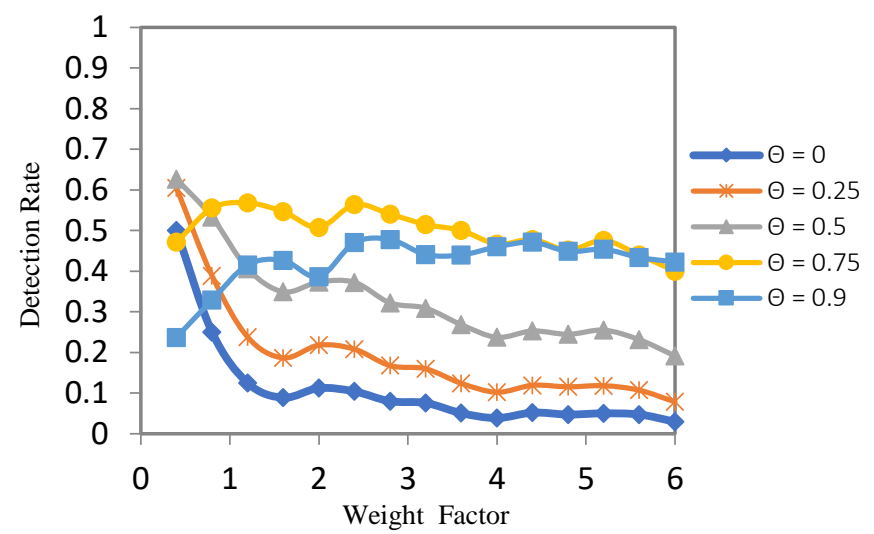

(a)

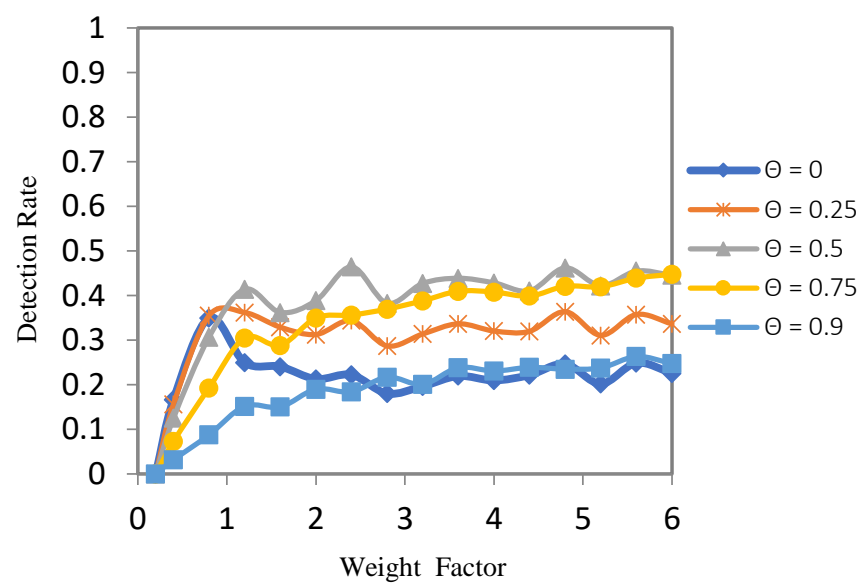

(b)

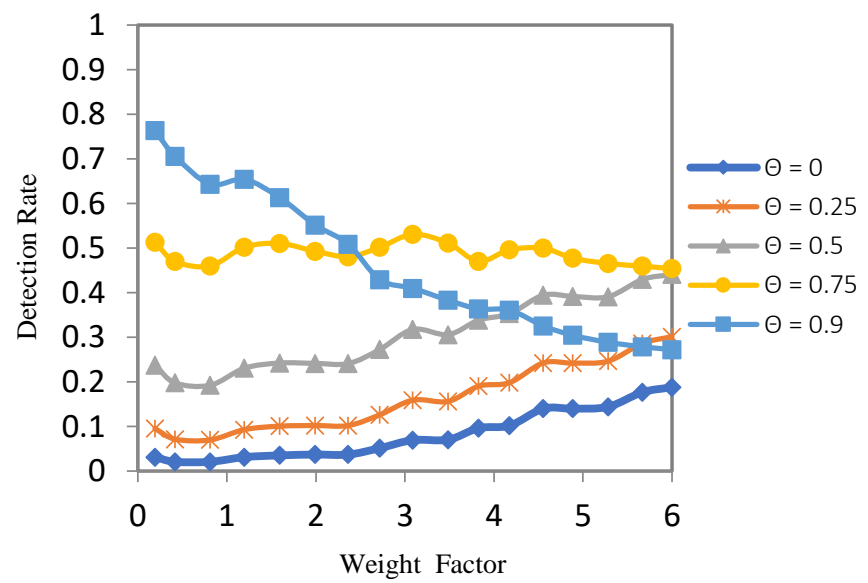

(c)

Fig. 7. Evaluation of $\min \Psi^{*}$ (a) $|A|=40$ (b) $|A|=100$ (c) $|A|=(2-80)$ 
In Fig. (6-7), $\bar{\Psi}^{*}$ and $\min \Psi^{*}$ increase initially and then decrease with increment in both $W_{F}$ and $\Theta$. Because the data overlapping minimizes initially and after a threshold it starts increasing during the allocation of dataset. It is also noticed that $\Theta=0.9$ shows distinct nature as chances of guessing the data are high instead of leaking it.

\section{CONCLUSION}

A malicious agent identification in cloud environment by exploiting the property of probability theory is discussed in this paper. Proposed model used the 2-level trees for the data allocation among various agents. These trees are utilized for the computation of various parameters for detection of guilty agent. The probability of detecting malicious agent, average success rate and detection rate are high even though weight factor and probability of guessing are high that shows the efficiency of model. Future efforts could be made to improve the security of the most sensitive information via considering the threshold value and complex threat model.

\section{REFERENCES}

[1] Buyya, R., Broberg, J., and Goscinski, A., "Cloud Computing: Principles and Paradigms", vol. 87, John Wiley \& Sons, 2010.

[2] Gupta, I. and Singh, A. K., "An Integrated Approach for Data Leaker Detection in Cloud Environment", Journal of Information Science and Engineering, 2019.

[3] Gupta, I. and Singh, A. K., "A Confidentiality Preserving Data Leaker Detection Model for Secure Sharing of Cloud Data using Integrated Techniques", 7th International Conference on Smart Computing and Communication Systems (ICSCC), IEEE, Sarawak, Malaysia, pp. 1-5, 2019.

[4] Forbes, "State of Enterprise Cloud Computing 2018", [online] Available: https://www.forbes.com/sites/louiscolumbus/2018/08/30/state-of-enterprise-cloud-computing2018/\#2e5312c2265e, August, 2018.

[5] Cheng, L., Liu, F., and Yao, D., "Enterprise Data Breach: Causes, Challenges, Prevention, and Future Directions", WIREs Data Mining and Knowledge Discovery, vol. 7, pp. 1-14, September/October 2017.

[6] Shu, X., Yao, D., and Bertino, E., "Privacy-Preserving Detection of Sensitive Data Exposure", IEEE Transactions on Information Forensics and Security, vol. 10, no. 5, pp. 1092-1103, May 2015.

[7] Shu, X., Zhang, J., Yao, D., and Feng, W.-C., "Fast Detection of Transformed Data Leaks", IEEE Transactions on Information Forensics and Security, vol. 11, no. 3, pp. 528-542, March 2016.

[8] Kaur, K., Gupta, I., and Singh, A. K., "A Comparative Study of the Approach Provided for Preventing the Data Leakage", International Journal of Network Security \& its Applications (IJNSA), vol. 9, no. 5, pp. 21-33, September 2017.

[9] Breach Level Index, "A Global Database of Public Data Breaches", [online] Available: https://www.gemalto.com/press/pages/data-breaches-compromised-4-5-billion-records-in-first-half-of2018.aspx, October, 2018.

[10] Shehab, M., Bertino, E., and Ghafoor, A., "Watermarking Relational Databases using Optimization-Based Techniques", IEEE Transactions on Knowledge and Data Engineering, vol. 20, no. 1, pp. 116-129, 2008.

[11] Backes, M., Grimm, N., and Kate, A., "Data Lineage in Malicious Environments", IEEE Transactions on Dependable and Secure Computing, vol. 13, no. 2, pp. 178-191, March/April 2016.

[12] Guo, Y., Au, O. C., Wang, R., Fang, L., and Cao, X., "Halftone Image Watermarking by Content Aware Double-Sided Embedding Error Diffusion", IEEE Transactions on Image Processing, vol. 27, no. 7, pp. 3387-3402, 2018.

[13] Mareen, H., Praeter, J. De, Wallendael, G. Van, and Lambert, P., "A Novel Video Watermarking Approach Based on Implicit Distortions", IEEE Transactions on Consumer Electronics, vol. 64, no. 3, pp. 250-258, August 2018.

[14] Liu, Z., Huang, Y., and Huang, J., "Patchwork-Based Audio Watermarking Robust Against Desynchronization and Recapturing Attacks", IEEE Transactions on Information Forensics and Security, vol. 14, no. 5, pp. 1171-1180, May 2019.

[15] Hu, D., Zhao, D., and Zheng, S., "A New Robust Approach for Reversible Database Watermarking with Distortion Control", IEEE Transactions on Knowledge and Data Engineering, vol. 31, no. 6, pp. 10241037, June 2019.

[16] Papadimitriou, P. and Garcia-Molina, H., "Data Leakage Detection", IEEE Transactions on Knowledge and Data Engineering, vol. 23, no. 1, pp. 51-63, January 2011.

[17] Gupta, I. and Singh, A. K., "A Probability Based Model for Data Leakage Detection using Bigraph", $7^{\text {th }}$ International Conference on Communication and Network Security (ICCNS-2017), ACM, Tokyo, Japan, 2017. 
[18] Gupta, I. and Singh, A. K., "A Probabilistic Approach for Guilty Agent Detection using Bigraph after Distribution of Sample Data", Procedia Computer Science, vol. 125, pp. 662-668, 2018.

[19] Gupta, I., and Singh, A. K., "Dynamic Threshold based Information Leaker Identification Scheme", Information Processing Letters, vol. 147, pp. 69-73, 2019.

[20] Gupta, I., Singh, N., and Singh, A. K., "Layer-based Privacy and Security Architecture for Cloud Data Sharing", Journal of Communication Software and Systems (JCOMSS), vol. 15, no. 2, pp. 173-185, 2019. 